\title{
The utility of a genetic kidney disease clinic employing a broad range of genomic testing platforms: experience of the Irish Kidney Gene Project
}

\author{
Elhussein A. E. Elhassan ${ }^{1,2}\left(\right.$ D . Susan L. Murray ${ }^{1,2} \cdot$ Dervla M. Connaughton $^{3,4} \cdot$ Claire Kennedy $^{1} \cdot$ Sarah Cormican $^{1}$. \\ Cliona Cowhig ${ }^{1}$. Caragh Stapleton ${ }^{5} \cdot$ Mark A. Little $^{6} \cdot$ Kendrah Kidd $^{7}$. Anthony J. Bleyer ${ }^{7} \cdot$ Martina Živná $^{8}$. \\ Stanislav Kmoch ${ }^{7,8}$ - Neil K. Fennelly ${ }^{9}$ Brendan Doyle ${ }^{9}$. Anthony Dorman ${ }^{9,10}$ - Matthew D. Griffin ${ }^{11,12}$. \\ Liam Casserly ${ }^{13}$. Peter C. Harris ${ }^{14}$. Friedhelm Hildebrandt ${ }^{15} \cdot$ Gianpiero L. Cavalleri $^{5} \cdot$ Katherine A. Benson $^{5}$. \\ Peter J. Conlon ${ }^{1,2}$
}

Received: 19 October 2021 / Accepted: 16 December 2021 / Published online: 31 January 2022

(c) The Author(s) 2022

\begin{abstract}
Background and aims Genetic testing presents a unique opportunity for diagnosis and management of genetic kidney diseases (GKD). Here, we describe the clinical utility and valuable impact of a specialized GKD clinic, which uses a variety of genomic sequencing strategies.

Methods In this prospective cohort study, we undertook genetic testing in adults with suspected GKD according to prespecified criteria. Over 7 years, patients were referred from tertiary centres across Ireland to an academic medical centre as part of the Irish Kidney Gene Project.

Results Among 677 patients, the mean age was of $37.2 \pm 13$ years, and $73.9 \%$ of the patients had family history of chronic kidney disease (CKD). We achieved a molecular diagnostic rate of 50.9\%. Four genes accounted for more than $70 \%$ of identified pathogenic variants: PKDI and PKD2 ( $n=186,53.4 \%), M U C 1$ (8.9\%), and COL4A5 (8.3\%). In 162 patients with
\end{abstract}

Katherine A. Benson and Peter J. Conlon contributed equally to this work.

Elhussein A. E. Elhassan

elhusseinelhassan@beaumont.ie

1 Department of Nephrology and Transplantation, Beaumont Hospital, Dublin, Ireland

2 Department of Medicine, Dublin, Royal College of Surgeons in Ireland, Dublin, Ireland

3 Schulich School of Medicine and Dentistry, University of Western Ontario, London, ON, Canada

4 Division of Nephrology, Department of Medicine, London Health Sciences Centre, London, ON, Canada

5 School of Pharmacy and Biomolecular Sciences, Royal College of Surgeons, Dublin, Ireland

6 Trinity Health Kidney Centre, Trinity Translational Medicine Institute, Trinity College Dublin, St James' Street, Dublin 8, Ireland

7 Section on Nephrology, Wake Forest School of Medicine, Winston-Salem, NC, USA

8 Research Unit for Rare Diseases, Department of Paediatrics and Inherited Metabolic Disorders, First Faculty of Medicine, Charles University in Prague, Prague, Czech Republic
9 Department of Pathology, Beaumont Hospital, Dublin, Ireland

10 Department of Pathology, Royal College of Surgeons in Ireland, Dublin, Ireland

11 Nephrology Department, Galway University Hospitals, Saolta University Healthcare Group, Galway, Ireland

12 Regenerative Medicine Institute (REMEDI) at CÚRAM Centre for Research in Medical Devices, School of Medicine, National University of Ireland, Galway, Ireland

13 Department of Nephrology and Internal Medicine, University Hospital Limerick, Limerick, Ireland

14 Division of Nephrology and Hypertension, Mayo Clinic, Rochester, MN, USA

15 Department of Paediatrics, Boston Children's Hospital, Harvard Medical School, Boston, MA 02115, USA 
a genetic diagnosis, excluding PKD1/PKD2, the a priori diagnosis was confirmed in 58\% and in $13 \%$ the diagnosis was reclassified. A genetic diagnosis was established in 22 (29.7\%) patients with CKD of uncertain aetiology. Based on genetic testing, a diagnostic kidney biopsy was unnecessary in 13 (8\%) patients. Presence of family history of CKD and the underlying a priori diagnosis were independent predictors $(P<0.001)$ of a positive genetic diagnosis.

Conclusions A dedicated GKD clinic is a valuable resource, and its implementation of various genomic strategies has resulted in a direct, demonstrable clinical and therapeutic benefits to affected patients.

\section{Graphical abstract}

\section{The utility of a genetic kidney disease clinic employing a broad range of genomic testing platforms: Experience of the Irish Kidney Gene Project}
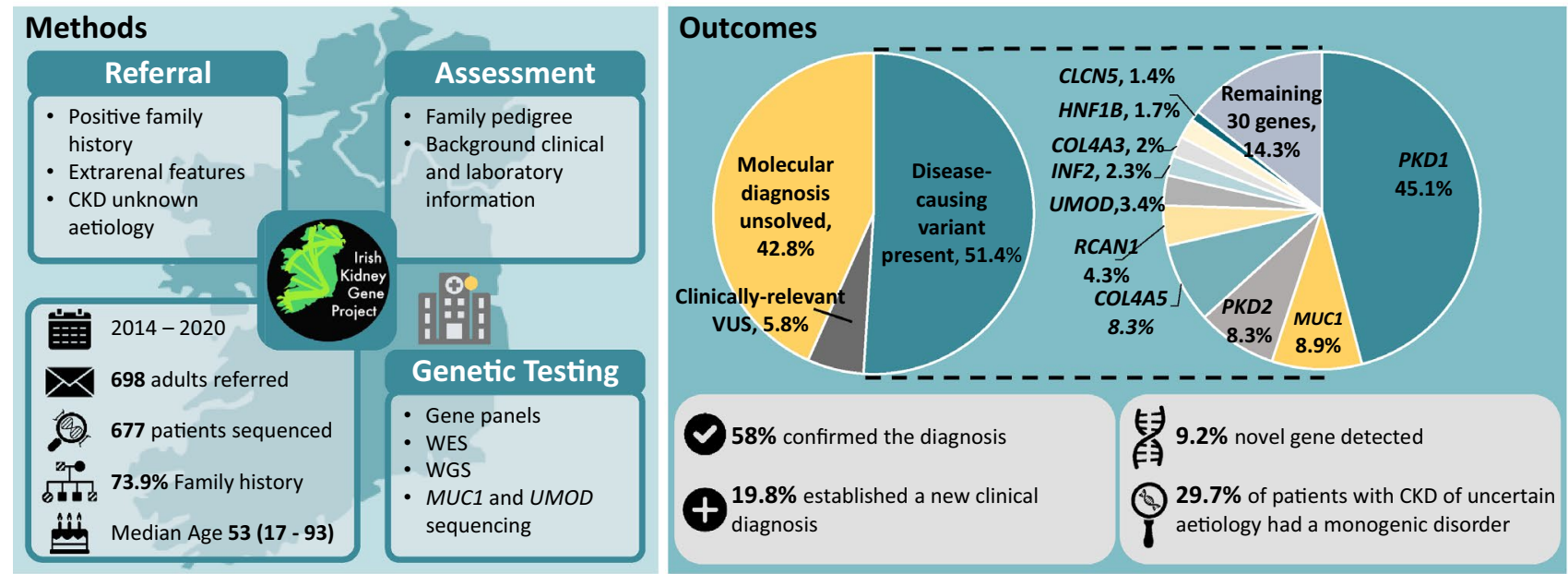

Elhassan EAE et al, 2021

CONCLUSION: In adult patients with suspected inherited kidney disease, genetic testing had a diagnostic yield exceeding 50\%, with clinical implications in many cases.

Keywords Chronic kidney disease $\cdot$ Inherited kidney diseases $\cdot$ Next-generation sequencing $\cdot$ Polycystic kidney genetics Genetic kidney disease

\section{Introduction}

Testing for genetic kidney diseases (GKD), encompasses an array of more than 150 rare monogenic disorders, uncovers new horizons for diagnosis and management of patients and their families [1, 2]. Up to $35 \%$ of adults with chronic kidney disease (CKD) report a positive family history, suggesting a hereditary element [3]. While a strong genetic component of certain forms of GKD such as autosomal dominant polycystic kidney disease (ADPKD) is well recognised, other forms of adult GKD have historically been overlooked, and equally can be complex and multifaceted $[4,5]$.

The establishment of a specialised genetics service, utilising a multidisciplinary team (MDT) of clinical nephrologists, clinical geneticists, genetics counsellors, nurses, pathologists, and research geneticists/bioinformaticians is warranted to diagnose, manage, and treat patients with
GKD [6]. Therefore, to determine the efficacy of genomic sequencing technologies, including next-generation sequencing (NGS), and the underlying genetic cause of disease in patients with suspected GKD, we established a research program known as the Irish Kidney Gene Project (IKGP) and an associated clinical service called the Genetic Kidney Disease Clinic (GKDC), at Beaumont Hospital, Dublin, Ireland [7, 8].

To diagnose GKD, several approaches can be adopted. Recent studies have focused on the utility of whole-exome sequencing (WES) in diagnosing GKD, with a diagnostic yield of $9-37 \%$ for monogenic disease depending on the patient population [9-12]. While WES is undoubtedly useful, it may not be practical or cost-efficient for all GKD clinics. Indeed, WES has been described as ineffective for diagnosis of ADPKD, the most common form of GKD [13]. NGS-based targeted gene panels may be 
considered as an alternative, with several studies reporting diagnostic rates ranging from 20 to $78 \%$ [14-16]. Other techniques may be required for specific genetic diseases. For example, the $M U C 1$ gene contains a highly repetitive region with a high guanosine/cytosine content, resulting in the inability of WES and NGS panels to identify $M U C l$ variants, one of the most common causes of autosomal dominant tubulointerstitial kidney disease (ADTKD) [17]. Specialized testing is required for this condition [18]. Finally, the use of WES or indeed whole genome sequencing (WGS) allows future-proofing of diagnostic tests, allowing for reanalysis of data as novel genes are discovered. Through integration with research centres, WES and WGS can be utilised to assist in the identification of novel GKD genes. Thus, a multi-faceted approach provides the best opportunity to supply genetic diagnoses.

In this prospective study, we describe our overall experience of the IKGP over the 7-year period from 2014 to 2020 and the clinical impact of GKDC on patients, including some patient subsets that have been previously described [11, 19-24].

\section{Methods}

\section{Patient data}

Adult patients attending a university-based academic Department of Nephrology, the Irish National Kidney Transplant Centre, at Beaumont Hospital, Dublin were recruited into this prospective cohort study. Ethical approval was sought and granted by the Ethics Review Board of Beaumont Hospital (REC 19/28). All patients gave explicit informed consent to participate.

Letters were sent out to nephrologists nationwide to inform them of the service and invite them to refer any adult patient (age $\geq 18$ years) with CKD who had either a positive family history, extrarenal features, or had CKD of "uncertain aetiology" (uCKD). Depending on the clinical and histological findings of the nephrologists' referrals, patients were grouped into seven categories of a priori clinical diagnoses. A detailed description of these categories and the diagnostic genomic methods used are listed in the Supplementary Material.

All patients referred to the GKDC, were reviewed and counselled by two among the following trained nephrologists-PC, CK, DC, SM, EE-with an interest in GKD and underwent research genetic testing guided by the a priori diagnosis.

\section{Genetic diagnosis}

The choice of genetic testing was guided by the patient's a priori diagnosis, the likely success of sequencing strategies and cost considerations. Sequencing and bioinformatics analyses for gene panels [19, 23], WES [11, 22, 25] and WGS [24] were performed by DMC, PCH, FH, GLC, $\mathrm{KAB}$ as described previously(see Supplementary Material). $M U C 1$ genotyping [18] was undertaken at the Broad Institute, while immunostaining for MUC1fs in urinary cell smears or kidney biopsy [20] followed by entire MUC1 sequencing using either Illumina [20] or PacBio Single Molecule, Real-Time (SMRT) Sequencing [26] were provided by Charles University in Prague by KK, AJB, MZ, SK. In each case these results of genomic testing were assessed by the MDT of clinical nephrologists with specific experience and training in GKD together with experts in clinical genetics and bioinformatics. Variants were prioritised and classified as per the American College of Medical Genetics (ACMG) guidelines [27].

Where a genetic diagnosis was made, patients were invited by the clinic to undergo a confirmatory genetic testing at an accredited clinical lab using a second sample, along with counselling on the implications of the results on their management and on other family members. This was required to ensure correct governance when including the test result in the clinical record and ensured complete accuracy of both the variant identification and interpretation. Only ACMG likely pathogenic/pathogenic results replicated at the clinical lab were returned to patients.

\section{Statistics}

Patient characteristics and genetic diagnosis were collected, and descriptive statistics were expressed as mean $\pm \mathrm{SD}$, percentages or median [interquartile range, IQR]. We evaluated clinical predictors favouring identification of a genetic diagnosis using logistic regression analyses. Data were analysed using STATA SE (version 16 StataCorp, College Station, TX, USA). Probability of a type 1 error less than 0.05 was statistically significant.

\section{Results}

\section{Cohort description}

A total of 698 affected adult individuals $(n=522$ families) were referred to the GKD service, none of whom had undergone prior genomic testing. Twenty-one patients were excluded from analysis as they declined participation, were deemed not to require testing upon referral assessment, or did not provide a sample for analysis (Fig. 1). The remaining 
Fig. 1 Flowchart of genetic kidney disease clinic recruitment, sequencing technologies, and outcome. IKGP Irish Kidney Gene Project, $M U C 1$ mucin 1 gene, MLPA multiplex ligationdependent probe amplification, $L R$ - $P C R$ long-range polymerase chain reaction, $U M O D$ Uromodulin, $V U S$ variant of uncertain significance, WES whole-exome sequencing, $W G S$ whole-genome sequencing

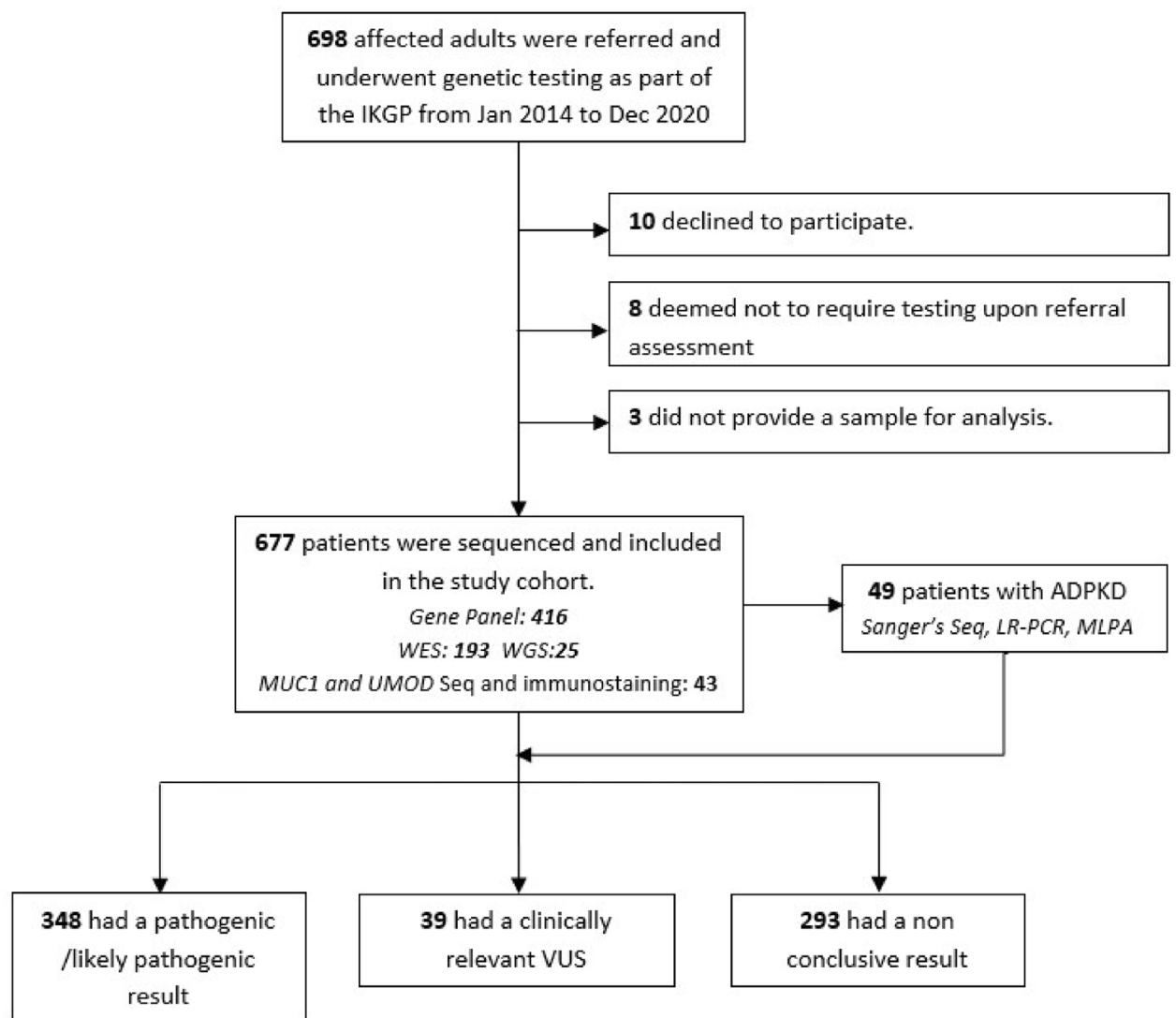

677 adults ( $n=501$ families) underwent genetic sequencing and formed the study cohort. Table 1 presents the baseline characteristics of the patients stratified by their genomic sequencing status.

The study population had a slight male preponderance, with 358 (52.9\%) males. One-hundred and eighteen (17.4\%) patients had disease onset at $<18$ years of age with a mean age of $10.2 \pm 5.7$ years, and $450(66.5 \%)$ patients presented as adults, with a mean age of $37.2 \pm 13$ years. Five hundred (73.9\%) participants reported a family history of renal disease $(P=<0.001)$. Sixty-five $\%$ of patients reached end stage kidney disease (ESKD) at the last review, with a median age at ESKD of 30 (interquartile range (IQR) 20-43) years. Two hundred fifteen $(31.8 \%)$ patients had CKD, defined as decreased estimated glomerular filtration rate lower than $60 \mathrm{ml} / \mathrm{min} / 1.73 \mathrm{~m}^{2}$ for 3 months or longer, by 47 (IQR 37-59) years of age. A total of 664 (98\%) were self-reported as Caucasian, and more than $95 \%$ of the cohort were Irish, which is representative of the Irish population [28]. None of the patients reported consanguinity.

According to the criteria adopted, we achieved a genetic diagnosis in $46.5 \%$ of the 501 families, corresponding to $51.4 \%$ of the 677 patients (Table 2, Supplementary Table S1). Two-thirds of patients with a reported family history of kidney disease achieved a genetic diagnosis $(67.2 \%$ $(336 / 500))$ versus $31 \%(43 / 140)$ in patients with no family history of kidney disease $(P=<0.001)$. Segregation analysis was required for 56 (9\%) families. Amongst the 40 identified monogenic disorders, ACMG pathogenic or likely-pathogenic variants within four genes accounted for $70.7 \%$ of all identified causative variants; $P K D I(n=157 / 348 ; 123$ families), PKD2 ( $n=29 / 348 ; 22$ families), MUC1 ( $n=31 / 348$; 10 families), and COL4A5 ( $n=29 / 348 ; 18$ families). The remaining $29.3 \%$ of patients with a genetic diagnosis contained variants across a further 36 genes (Figs. 2 and 3).

In addition, variants of uncertain significance (VUS) considered to be clinically interesting by the MDT were detected in $11.8 \%$ (39/329; 32 families) of patients without a diseasecausing variant. Segregation analysis is underway in 29 of these VUS families in an effort to reclassify these variants. In the remaining 236 families ( $47.1 \%$ ), we were not able to obtain a genetic diagnosis.

\section{A priori diagnosis and identification of causative variants}

Polycystic kidney disease (PKD): PKD was the most prevalent a priori diagnosis $(241 / 677,35.6 \%)$. The a priori clinical diagnoses are listed in Table 1, with a detailed description in Supplementary Material. We identified a disease-causing variant in $78.7 \%$ (148/188) of families recruited with a priori diagnosis of PKD (Table 2), and targeted gene-panels were 
Table 1 Characteristics of the 677 affected individuals (501 families) sequenced by the Irish Kidney Gene Project (IKGP)

\begin{tabular}{|c|c|c|c|c|}
\hline Characteristics & $\begin{array}{l}\text { Total } \\
\text { sequenced } \\
(n=677)\end{array}$ & $\begin{array}{l}\text { Total vari- } \\
\text { ants identified } \\
(n=387)\end{array}$ & Unsolved $(n=290)$ & $P$ value \\
\hline \multicolumn{5}{|l|}{ A priori clinical diagnosis, $n(\%)$} \\
\hline PKD & $241(35.6)$ & $205(53)$ & $36(12.4)$ & \multirow[t]{7}{*}{$<0.001$} \\
\hline CAKUT & $85(12.6)$ & $12(3.1)$ & $73(25.2)$ & \\
\hline Chronic GN & $112(16.5)$ & $24(6.2)$ & $88(30.3)$ & \\
\hline TIKD & $75(11.1)$ & $49(12.7)$ & $26(9)$ & \\
\hline AS/FSGS & $72(10.6)$ & $57(14.7)$ & $15(5.2)$ & \\
\hline Others & $18(2.7)$ & $13(3.3)$ & $5(1.7)$ & \\
\hline $\mathrm{uCKD}$ & $74(10.9)$ & $27(7)$ & $47(16.2)$ & \\
\hline \multicolumn{5}{|l|}{ Recruited from, $n(\%)^{1}$} \\
\hline Monogenic kidney disease study & $138(20.4)$ & $56(14.5)$ & $82(28.3)$ & \multirow[t]{3}{*}{$<0.001$} \\
\hline PKD study & $208(30.7)$ & $177(45.7)$ & $31(10.7)$ & \\
\hline GKD clinic & $331(7.8)$ & $154(39.8)$ & $177(61)$ & \\
\hline Median age, yrs (range) & $53(18-93)$ & $54(18-88)$ & $51(18-93)$ & 0.018 \\
\hline \multicolumn{5}{|l|}{ Age in years at onset of disease, $n(\%)$} \\
\hline$<18$ (childhood onset) & $118(17.4)$ & $66(17.1)$ & $52(17.9)$ & \multirow[t]{3}{*}{0.142} \\
\hline$\geq 18$ (adult onset) & $450(66.5)$ & $274(70.8)$ & $176(60.7)$ & \\
\hline Unavailable & $109(16.1)$ & $47(12.1)$ & $62(21.4)$ & \\
\hline \multicolumn{5}{|l|}{$\mathrm{ESKD}, n(\%)$} \\
\hline Yes & $440(65)$ & $228(58.9)$ & $212(73.1)$ & \multirow[t]{3}{*}{0.001} \\
\hline No & $215(31.8)$ & $142(36.7)$ & $73(25.2)$ & \\
\hline Missing & $22(3.2)$ & $17(4.4)$ & $5(1.7)$ & \\
\hline Median age at onset of ESKD, [IQR] & $30[20-43]$ & 30 [21-44] & 30 [18-43] & 0.425 \\
\hline \multicolumn{5}{|l|}{ Sex } \\
\hline Male & $358(52.9)$ & $196(50.6)$ & $162(55.8)$ & \multirow[t]{2}{*}{0.163} \\
\hline Female & $319(47.1)$ & $191(49.4)$ & $128(44.2)$ & \\
\hline \multicolumn{5}{|l|}{ FHx of CKD, $n(\%)^{2}$} \\
\hline Yes & $500(73.9)$ & $336(86.8)$ & $164(56.6)$ & \multirow[t]{3}{*}{$<0.001$} \\
\hline No & $140(20.7)$ & $43(11.1)$ & $97(33.4)$ & \\
\hline Unavailable & $37(5.4)$ & $8(2.1)$ & $29(10)$ & \\
\hline \multicolumn{5}{|l|}{ Self-reported ethnicity } \\
\hline Irish & $646(95.4)$ & $373(96.4)$ & $273(94.2)$ & \multirow[t]{4}{*}{0.551} \\
\hline Other Europeans & $18(2.7)$ & $8(2.1)$ & $10(3.4)$ & \\
\hline Black & $8(1.2)$ & $4(1)$ & $4(1.4)$ & \\
\hline Asian & $5(0.7)$ & $2(0.5)$ & $3(1)$ & \\
\hline
\end{tabular}

$A S$ Alport syndrome, CAKUT congenital anomalies of the kidney and urinary tract, $C K D$ Chronic kidney disease, ESKD end stage kidney disease, GKD genetic kidney disease, IQR Interquartile range, $F H x$ family history, FSGS focal segmental glomerulosclerosis, $G N$ glomerulonephritis, $P K D$ polycystic kidney disease, $u C K D$ CKD of uncertain aetiology, TIKD tubulointerstitial kidney disease, Yrs years

${ }^{1}$ In total, 677 patients were reviewed in the genetic kidney disease (GKD) clinic, recruited and bio-banked for the evaluation and management of nephropathy. Included in this large cohort, two groups of patients were previously published in the Monogenic Kidney Disease Study [11] and PKD study [23]; these are grouped separately for clarity

${ }^{2} \mathrm{~A}$ positive family history of kidney disease that was reported by the patient (either a 1st-degree relative (parent, child, or sibling) or a 2nd -degree relative (grandparent, aunt, uncle, niece, nephew, or cousin) used as the primary sequencing technology. In $191 \mathrm{PKD}$ patients with ACMG likely pathogenic/pathogenic variants, 157 carried a disease-causing variant in $P K D 1$, and 29 in PKD2 (Supplementary Table S1). Of the remaining five patients, three patients were found to have $P K H D 1$ variants associated with autosomal recessive PKD (ARPKD), and two carried $M A P 2 K 2$ variants associated with cardio-faciocutaneous syndrome. Ten PKD families were identified to harbour clinically relevant VUS, whereas 30 families had no diagnostic results (16\%). 


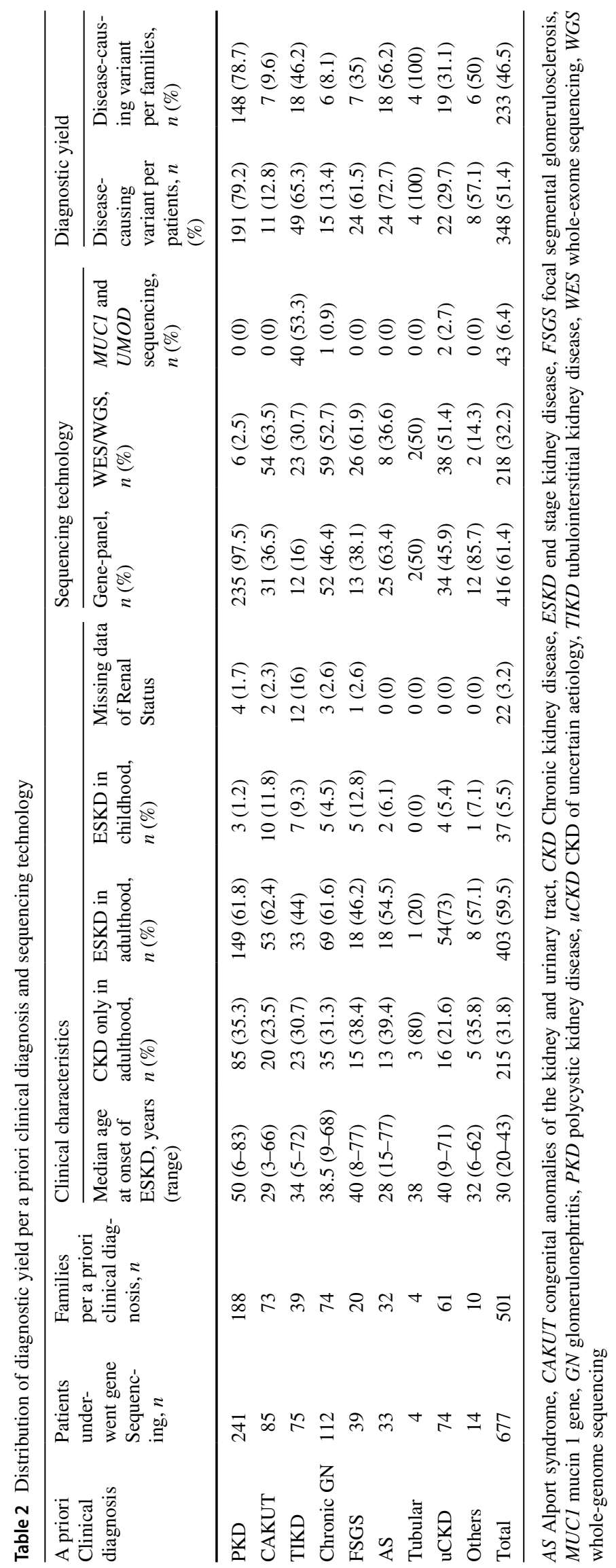




\section{A Adult participants sequenced by the IKGP $(n=677)$}

\section{$\square$ Disease-causing variant present \\ $\square$ Clinicaly-relevant vus \\ $\square$ Molecular diagnosis unsolved}

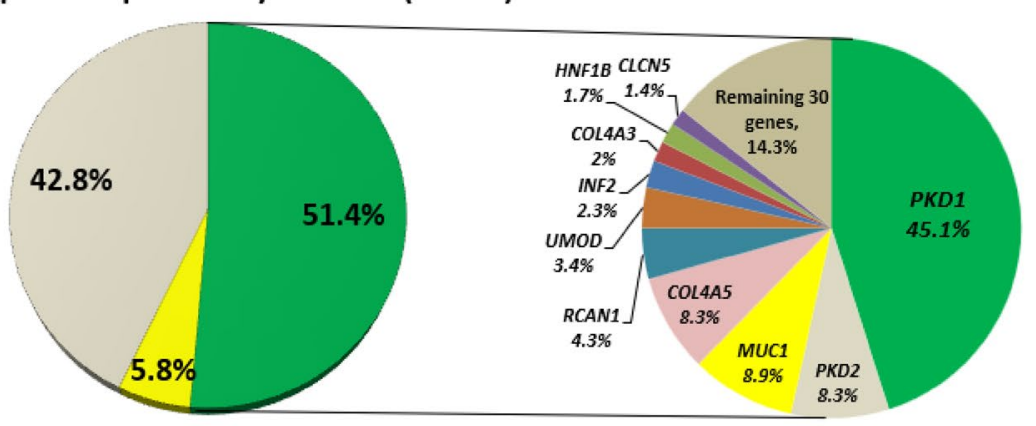

B

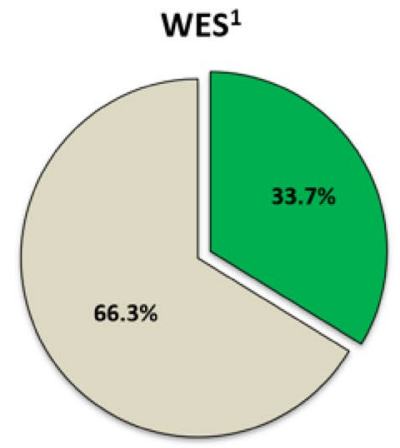

Fig. 2 a Breakdown of disease-causing genes containing a pathogenic variant detected by the Irish Kidney Gene Project in whole cohort. b Pathogenic detection rate in whole-exome sequencing (WES) and a targeted gene panel. IKGP Irish Kidney Gene Project, VUS variant

Non-Cystic GKD: Among 313 families with non-cystic GKD, the diagnostic yield was $27.1 \%$. The diagnostic yield varied within each diagnostic subgroup (Table 2, Supplementary Fig. S1).

Alport syndrome (AS)/focal segmental glomerulosclerosis (FSGS): Within the AS/FSGS cohort, we identified ACMG pathogenic/likely pathogenic variants within seven genes accounting for 48/72 individuals (66.7\%). COL4Arelated variants $(n=24)$ were the most frequent in this patient group and COLAA5 predominated $(n=20,41.6 \%)$. In one large family with autosomal dominant FSGS, we discovered a heterozygous NM_004414:p.Ile162Thr RCAN1 variant, responsible for the patients' FSGS [24]. Eight patients (3 families) were identified with disease-causing INF2 variants, with a clear positive family history of proteinuric renal disease.

Glomerulonephritis (GN) and IgA Nephropathy: We identified a genetic diagnosis in 6 out of 74 families referred with chronic GN (8.1\%). We detected three variants segregating in families with IgA nephropathy as reported by Stapleton et al. [22]. We did not identify a genetic diagnosis in any families with MPGN/C3GN. In family F87, the proband presented with advanced CKD stage 5, proteinuria and family history of CKD. Following re-examination of

\begin{tabular}{l|c}
\hline \multicolumn{1}{c}{ Genes } & No of Varients \\
\hline PKD1 & 157 \\
\hline PKD2 & 29 \\
\hline MUC1 & 31 \\
\hline COL4A5 & 29 \\
\hline RCAN1 & 15 \\
\hline UMOD & 12 \\
\hline INF2 & 8 \\
\hline COL4A3 & 7 \\
\hline HNF1B & 6 \\
\hline CLCN5 & 5 \\
\hline NPHP1 & 5 \\
\hline NPHS2 & 4 \\
\hline PKHD1 & 3 \\
\hline PAX2 & 3 \\
\hline C3 & 4 \\
\hline IFT140 & 2 \\
\hline CLCNKB & 2 \\
\hline 23 other genes & 26 \\
\hline
\end{tabular}

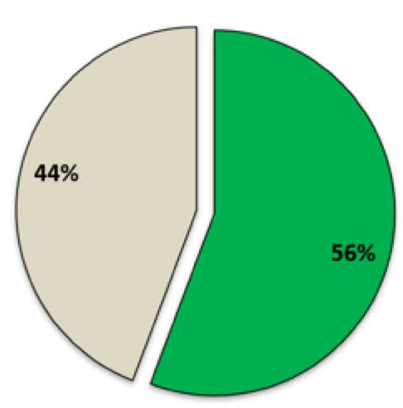

of unknown significance. ${ }^{1}$ More than 475 known chronic kidney disease genes; see references $[11,25] .{ }^{2}$ Roche SeqCap EZ Choice (227 genes panel) and Roche NimbleGen HeatSeq panel (11 genes panel); see reference [23]

the kidney biopsy specimen, immunostaining for MUC1fs in urinary cell smears was positive confirming the diagnosis of MUC1-ADTKD, which was not suspected on clinical grounds before this study, hence correcting the clinical diagnosis from GN to ADTKD. Proteinuria was thought to be related to chronic changes.

Tubulointerstitial kidney disease (TIKD): The well-established $M U C 1$ cytosine duplication variant was detected in 6 families (25 patients) with ADTKD. In four ADTKD families (7 patients) where a $M U C l$ variant was not identified, we used non-invasive immunohistochemical urinary smear or kidney biopsies to confirm the presence of the frameshifted MUC1 protein (MUC1fs). Alternative genetic diagnoses were made in seven ADTKD families (18 patients); five with ADTKD-UMOD (12 patients), and two with ADTKD$H N F 1 B$ (6 patients). Amongst ADTKD families, TIKD predominated as an a priori diagnosis, but eight patients were initially referred with uCKD $(n=4)$, congenital anomalies of the kidney and urinary tract (CAKUT) $(n=3)$, or GN $(n=1)$. WES identified disease-causing variants in further eight patients with a priori diagnosis of TIKD and inconclusive biopsy findings.

$u C K D$ : Amongst 61 families (74 patients) referred with a priori diagnosis of uCKD, 31.1\% (19/61; 22 patients) were 


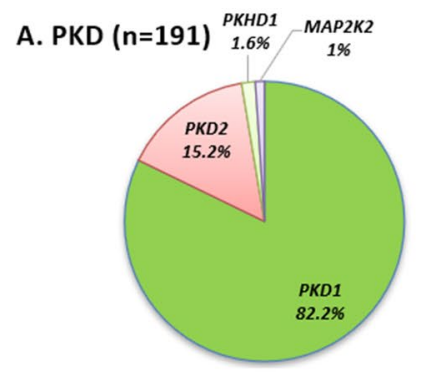

E. GN $(n=15)^{1}$

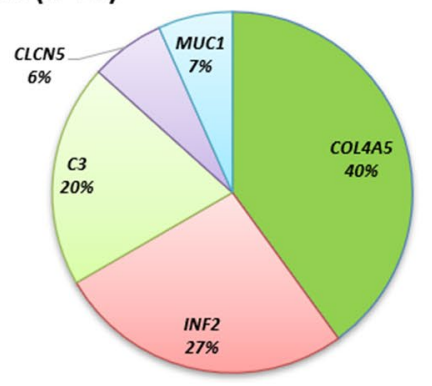

B. TIKD $(n=49) \quad$ IFT140 $\underset{2 \%}{\text { BBS9 }}$

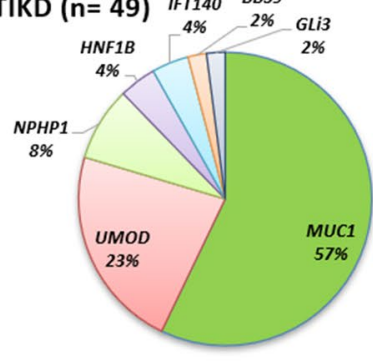

F. Others $(n=12)$

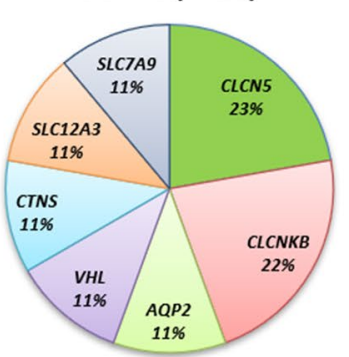

C. CAKUT $(n=11)$

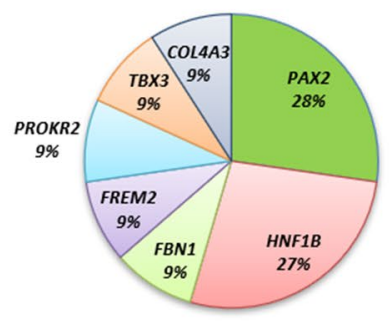

D. AS/FSGS $(\mathrm{n}=48)$ FANC1

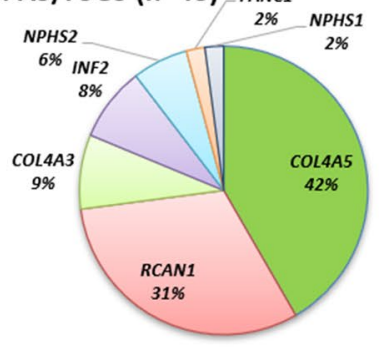

G. uCKD ( $n=22)$

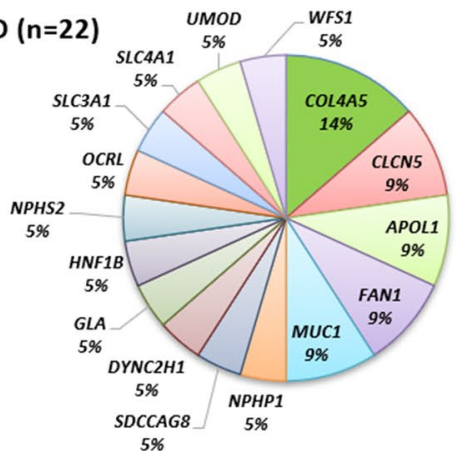

Fig. 3 Disease-causing genes detected in the IKGP participants separated according to a priori diagnosis. CAKUT congenital anomalies of the kidney and urinary tract, $A S / F S G S$ Alport syndrome/focal segmental glomerulosclerosis, $G N$ glomerulonephritis, $P K D$ polycystic kidney disease, $u C K D$ chronic kidney disease of uncertain aetiology, TIKD tubulointerstitial kidney disease. ${ }^{1}$ In family $\mathrm{F} 87$, the proband

found to have a known monogenic disorder, bringing their diagnostic odyssey to an end, and emphasising the difficulties in making a clinical diagnosis in these very rare conditions without genetics support (Supplementary Table S1). In patients with a priori diagnosis of CAKUT, we detected 11 pathogenic/likely pathogenic variants in 11 individuals, corresponding to 7 of 73 families $(9.6 \%)$. WES on three families with a child who had prune-belly syndrome did not reveal any underlying genetic cause.

Kidney donors: Three potential live kidney donors attended the clinic for screening due to a strong family history of CKD. In one case the potential donor was the sister of a patient with documented ADTKD-MUC1, while the other two patients had siblings with AS. In each case, we were able to confirm that the potential donors did not carry the disease-causing variants and were able to progress with living donation assessment.

\section{Diagnostic yield per platform and their clinical utility}

WES resulted in a diagnostic rate of approximately $34 \%$, whereas gene panel sequencing resulted in 56\% (Fig. 2B). In ADTKD families, $M U C 1$ genotyping and targeted gene panel testing for $U M O D, R E N$ and $H N F 1 B$ at the Broad resulted in a diagnostic rate of $70 \%$. The further addition of had initial presentation of CKD stage 5, proteinuria, and family history of CKD, and referred with a priori diagnosis of GN. Proteinuria is thought to be a result of chronic changes rather than representing nature of the primary disease tubulointerstitial disease following the establishment of genetic diagnosis by $M U C 1$ sequencing and immunostaining for MUC1fs

urine smear analysis and tissue immunostaining for MUC1fs followed by sequencing of the entire $\mathrm{MUCl}$ gene increased this to $86 \%$ in ADTKD patients.

Excluding ADPKD cases, genetic testing confirmed the a priori diagnosis in 58\% of individuals. Patients' diagnoses were refined or a new diagnosis was made in $13 \%$ and $20 \%$ of non-ADPKD patients, respectively. In 15 of the 162 patients (9\%), a new gene was identified as had been reported by Lane et al. [24] (Table 3). A genetic diagnosis facilitated a change in treatment plan in 28/162 (17.3\%) patients, while a diagnostic kidney biopsy was deemed unnecessary in 13/162 (8\%) patients as a direct result of a genetic diagnosis. In non-ADPKD patients, genetic results prompted reverse phenotyping such as targeted work-up for other associated extra-renal conditions in 51/162 (31.5\%) and a further 70/162 (43.2\%) patients had appropriate familial cascade testing.

Clinical lab validation of a genetic diagnosis was undertaken in 89/131 eligible patients, excluding those with ADPKD. Five did not return for clinical validation and 37 patients are awaiting return of results at the time of submission. At the beginning of the project, the median time from being evaluated in GKDC to return of validated results was 570.5 (IQR 317-1385) days, though the turnaround time decreased to 131 (IQR 100-205) days over the last 12 months of the study. 
Table 3 Summary of clinical outcomes of 162 patients (excluding cystic kidney disease) with a confirmed pathogenic diagnosis and the impact of genomic diagnosis on subsequent treatment

\begin{tabular}{ll}
\hline Diagnostic utility, $n(\%)$ & \\
Confirmed the a priori diagnosis & $94(58)$ \\
Refined the a priori diagnosis* & $21(13)$ \\
Established a new diagnosis & $32(19.8)$ \\
Novel candidate gene identified & $15(9.2)$ \\
Clinical utility, $n(\%)$ & \\
Negate Biopsy based on genomic diagnosis & $13(8)$ \\
Cascade tests/family counselling & $70(43.2)$ \\
Change pharmacological treatment & $28(17.3)$ \\
Additional assessment ordered to clarify extra-renal & $51(31.5)$ \\
$\quad$ features-reverse phenotyping & \\
\hline
\end{tabular}

*Genetic testing corrected/reclassified the a prior clinical diagnosis

\section{Factors favouring diagnostic outcome}

In multivariate logistic regression analysis, patients with causative variants were more than three-fold more likely to report family history of CKD (Odds ratio (OR) 3.69; 95\% confidence interval $(\mathrm{CI})=2.1-6.48 ; P=<0.001)$. Patients with the underlying a priori diagnosis of PKD (OR: 14.9; 95\% CI $=8.04-27.6 ; P=<0.001$ ), TIKD (OR $4.7 ; 95 \%$ CI $2.15-10.3 ; P=<0.001)$, AS (OR $24.6 ; 95 \%$ CI 6.4-93.1; $P=<0.001$ ), FSGS (OR 6.3; 95\% CI 2.3-17.4; $P=<0.001$ ), and UCKD (OR 7.6; 95\% CI 1.7-32.4; $P=<0.001)$ had a significantly higher frequency of diagnostic outcome relative to patients with GN. No statistical difference was observed regarding patients' age $(P=0.246)$, age at disease onset $<18$ years $(P=0.376)$, or sex $(P=0.471)$ between cases with or without diagnostic variants (Supplementary Tables S2 and S3).

\section{Discussion}

Our study outlines the complexity of monogenic disorders, and the advantages of using genomic testing from diagnostic and clinical perspectives. We identified disease-causing variants in 46.5\% (233/501) of GKD families (51.4\% (348/677) of patients). The diagnostic yield in our cohort was consistent with several earlier studies [5, 14, 15], but higher than other studies $[9,10]$. There are several factors that would explain our relatively high yield. First, we used a variety of genetic techniques to obtain a diagnosis. Earlier studies did not perform specialised genetic analysis for $M U C 1$ variants, which contributed to a significant number $(8.9 \%)$ of diagnoses in our population. We also used specific clinical criteria to limit our population to a group of patients with a high risk of familial kidney disease. Similar to other studies $[3,9-11,15]$, the presence of family history of CKD and the underlying a priori diagnosis were the two most significant predictors of a genetic diagnosis. In addition, a large proportion of the studied patients had clinically suspected PKD, which is known to have a high rate of genetic diagnosis. A targeted gene panel [23], designed to achieve high coverage of $P K D 1$ and $P K D 2$ was utilised in patients with an a priori diagnosis of PKD, which had considerable diagnostic utility (78.7\%) in this population. However, studies have reported higher diagnostic rates at 86-94\% [29-31], yet our relatively low diagnostic rate can be justified by the broad a priori definition which we adopted, in whom one-fifth of our patients reported no family history.

Employing WES, the diagnostic yield of around 34\% in our adult cohort was comparable with a recent WES study by Jayasinghe et al. [9], which reported a genetic diagnosis in 80/204 (39\%) patients across a spectrum of renal phenotype subcategories. In contrast to our study which exclusively involved adult patients, around $40 \%$ of the patients reported by Jayasinghe et al. were $<18$ years of age.

Up to $10 \%$ of total solved cohort had a COL4-related genetic diagnosis, a prominent, yet often unsuspected cause of GKD in adults, which correlated with high diagnostic yield in the AS cohort (56.2\%). A diagnostic yield of $35 \%$ was obtained using genomic sequencing in the FSGS cohort. Several large studies of adult patients with primary FSGS achieved diagnostic yields ranging from 29 to $37 \%$ and $12-30 \%$ for familial [32] and sporadic [32, 33] cases, respectively.

In Ireland, ADTKD accounts for $0.5 \%$ of ESKD patients [21]. The genetic diagnosis of ADTKD was made in a high percentage in our cohort. The ability to identify $M U C l$ variants was important for the evaluation of GKD in our cohort, using both $M U C 1$ genetic sequencing and newer techniques to detect the mutant MUC1fs protein.

The ultimate goal of genetic testing is the potential for personalised medicine. We validated and returned most of the research-based testing [34]. In adults, similar to previous studies [9-12], our data demonstrate that GKDC results provide a precise genetic diagnoses with diagnostic and therapeutic implications (Table 3 ). Genetic testing has numerous other advantages including prognostics and ruling in or out familial kidney donors [35].

A primary weakness of this study was the observational nature of the methodology. A mono-ethnic cohort could limit the generalizability of the results. Also, we cannot exclude potential selection bias by having PKD as the main a priori diagnosis and using specific clinical criteria that limit our cohort to a highly specified group. Lastly, no licensed genetic counsellors or clinical geneticists were involved in reviewing our patients. 


\section{Conclusions}

In this large prospective cohort, the usage of various genomic testing strategies demonstrates their clinical application value, with a diagnostic yield over $50 \%$ supporting the advantageous clinical and therapeutic impact in adult patients with GKD. In our experience, an active renal genetic service requires a variety of genomic strategies and an integrated collaboration between clinical nephrologists and geneticists.

Supplementary Information The online version contains supplementary material available at https://doi.org/10.1007/s40620-021-01236-2.

Acknowledgements We thank all patients who participated in this study and their physicians. We acknowledge that some of this sequencing was made possible from funding provided by the Punchestown Kidney Research Fund (EPSPD/2019/213) and the contribution of the Irish Kidney association/Health Research Board under the HRCI-HRB Joint funding scheme (Grant code: HRCI-HRB-2020-032).

Author contributions EE, SLM, DMC, CK, CS, MAL, KK, AJB, MZ, SK, KAB, GLC, PJC conceived and/or designed the work that led to the submission, acquired data and/or played an important role in interpreting the results; drafted or revised the manuscript. EE, SLM, DMC, CK, SC, CC, CS, MAL, KK, AJB, MZ, SK, NKF, BD, AD, MDG, LC, PCH, FH, GLC, KAB, PJC approved the final version. Each author contributed important intellectual content during manuscript drafting or revision, accepts personal accountability for the author's own contributions and agrees to ensure that questions pertaining to the accuracy or integrity of any portion of the work are appropriately investigated and resolved. The results presented in this article have not been published previously in whole or part, except in abstract form.

Funding Open Access funding provided by the IReL Consortium.

\section{Declarations}

Conflict of interest E.E reports funds by the Royal College of Surgeons in Ireland Blackrock Clinic StAR MD. S.L.M. reports educational grant from Amgen. D.M.C. was supported by funding from the Health Research Board, Ireland (HPF-2016-1674), and received International Pediatric Research Foundation Early Investigators' Exchange Program Award and Irish Nephrology Society Amgen Specialist Registrar Research Bursary. F.H. is the William E. Harmon Professor of Paediatrics. This research is supported by a grant from the National Institutes of Health to FH (5R01DK076683-13). MŽ and SK were supported by the Ministry of Health of the Czech Republic (Grant NU2107-00033), the Ministry of Education of the Czech Republic (Grant LTAUSA19068) and by institutional programs of Charles University in Prague (UNCE/MED/007 and PROGRES-Q26/LF1). The National Center for Medical Genomics (LM2018132) kindly provided MUC1 sequencing. CK, SC, CC, CS, MAL, KK, AJB, MZ, SK, NKF, BD, $\mathrm{AD}, \mathrm{MDG}, \mathrm{LC}, \mathrm{PCH}, \mathrm{FH}, \mathrm{GLC}, \mathrm{KAB}$, and PJC have nothing to disclose.

Ethical approval Ethical approval was sought and granted by the EthicsReview Board of Beaumont Hospital (REC 19/28).

Informed consent All patients gave explicit informed consent to participate.
Open Access This article is licensed under a Creative Commons Attribution 4.0 International License, which permits use, sharing, adaptation, distribution and reproduction in any medium or format, as long as you give appropriate credit to the original author(s) and the source, provide a link to the Creative Commons licence, and indicate if changes were made. The images or other third party material in this article are included in the article's Creative Commons licence, unless indicated otherwise in a credit line to the material. If material is not included in the article's Creative Commons licence and your intended use is not permitted by statutory regulation or exceeds the permitted use, you will need to obtain permission directly from the copyright holder. To view a copy of this licence, visit http://creativecommons.org/licenses/by/4.0/.

\section{References}

1. Devuyst $\mathrm{O}$ et al (2014) Rare inherited kidney diseases: challenges, opportunities, and perspectives. Lancet 383(9931):1844-1859

2. Soliman NA (2012) Orphan kidney diseases. Nephron Clin Pract 120(4):c194-c199

3. Connaughton DM et al (2015) The Irish Kidney Gene projectprevalence of family history in patients with kidney disease in Ireland. Nephron 130(4):293-301

4. Cocchi E, Nestor JG, Gharavi AG (2020) Clinical genetic screening in adult patients with kidney disease. Clin J Am Soc Nephrol 15(10):1497-1510

5. Thomas CP et al (2020) Initial experience from a renal genetics clinic demonstrates a distinct role in patient management. Genet Med 22(6):1025-1035

6. Mallett A et al (2016) A multidisciplinary renal genetics clinic improves patient diagnosis. Med J Aust 204(2):58-59

7. IKGP. The Irish Kidney Gene Porject. 2021; Available from: http://www.beaumont.ie/kidneycentre-aboutus-irishkidneygene project. Cited 10 Dec 2021

8. HSE. National Service Plan 2020. 2020; Available from: https:// www.hse.ie/eng/services/publications/national-service-plan-2020. pdf. Cited 21 May 2021

9. Jayasinghe $\mathrm{K}$ et al (2021) Clinical impact of genomic testing in patients with suspected monogenic kidney disease. Genet Med 23(1):183-191

10. Groopman EE et al (2019) Diagnostic utility of exome sequencing for kidney disease. N Engl J Med 380(2):142-151

11. Connaughton DM et al (2019) Monogenic causes of chronic kidney disease in adults. Kidney Int 95(4):914-928

12. Lata $\mathrm{S}$ et al (2018) Whole-exome sequencing in adults with chronic kidney disease: a pilot study. Ann Intern Med 168(2):100-109

13. Ali $\mathrm{H}$ et al (2019) PKD1 duplicated regions limit clinical utility of whole exome sequencing for genetic diagnosis of autosomal dominant polycystic kidney disease. Sci Rep 9(1):4141

14. Bullich $\mathrm{G}$ et al (2018) A kidney-disease gene panel allows a comprehensive genetic diagnosis of cystic and glomerular inherited kidney diseases. Kidney Int 94(2):363-371

15. Domingo-Gallego A et al. (2021) Clinical utility of genetic testing in early-onset kidney disease: seven genes are the main players. Nephrol Dial Transplant

16. Mansilla MA et al (2021) Targeted broad-based genetic testing by next-generation sequencing informs diagnosis and facilitates management in patients with kidney diseases. Nephrol Dial Transplant 36(2):295-305

17. Eckardt K-U et al (2015) Autosomal dominant tubulointerstitial kidney disease: diagnosis, classification, and management-a KDIGO consensus report. Kidney Int 88(4):676-683 
18. Blumenstiel B et al (2016) Development and validation of a mass spectrometry-based assay for the molecular diagnosis of Mucin-1 kidney disease. J Mol Diagn 18(4):566-571

19. Murray SL et al (2020) Utility of genomic testing after renal biopsy. Am J Nephrol 51(1):43-53

20. Živná M et al (2018) Noninvasive immunohistochemical diagnosis and novel MUC1 mutations causing autosomal dominant tubulointerstitial kidney disease. J Am Soc Nephrol 29(9):2418-2431

21. Cormican $S$ et al (2019) Autosomal dominant tubulointerstitial kidney disease (ADTKD) in Ireland. Ren Fail 41(1):832-841

22. Stapleton $\mathrm{CP}$ et al (2020) An exome sequencing study of 10 families with IgA nephropathy. Nephron 144(2):72-83

23. Benson KA et al (2021) The genetic landscape of polycystic kidney disease in Ireland. Eur J Hum Genet 29(5):827-838

24. Lane BM et al (2021) A rare autosomal dominant variant in regulator of calcineurin type 1 (RCAN1) gene confers enhanced calcineurin activity and may cause FSGS. J Am Soc Nephrol 32:1682-1695

25. CeGAT (2020)

26. Vylet'al P et al (2021) Plasma mucin-1 (CA15-3) levels in autosomal dominant tubulointerstitial kidney disease due to MUC1 mutations. Am J Nephrol 52:1-10

27. Richards $S$ et al (2015) Standards and guidelines for the interpretation of sequence variants: a joint consensus recommendation of the American College of Medical Genetics and Genomics and the Association for Molecular Pathology. Genet Med 17(5):405-424
28. Central Statistics Office (2021) Census 2016 results

29. Tan YC et al (2009) Novel method for genomic analysis of PKD1 and PKD2 mutations in autosomal dominant polycystic kidney disease. Hum Mutat 30(2):264-273

30. Cornec-Le Gall E et al (2013) Type of PKD1 mutation influences renal outcome in ADPKD. J Am Soc Nephrol 24(6):1006-1013

31. Rossetti $S$ et al (2007) Comprehensive molecular diagnostics in autosomal dominant polycystic kidney disease. J Am Soc Nephrol 18(7):2143-2160

32. Wang $\mathrm{M}$ et al (2019) Contributions of rare gene variants to familial and sporadic FSGS. J Am Soc Nephrol 30(9):1625-1640

33. Gribouval $\mathrm{O}$ et al (2018) Identification of genetic causes for sporadic steroid-resistant nephrotic syndrome in adults. Kidney Int 94(5):1013-1022

34. Jarvik GP et al (2014) Return of genomic results to research participants: the floor, the ceiling, and the choices in between. Am J Hum Genet 94(6):818-826

35. Stokman MF et al (2016) The expanding phenotypic spectra of kidney diseases: insights from genetic studies. Nat Rev Nephrol 12(8):472-483

Publisher's Note Springer Nature remains neutral with regard to jurisdictional claims in published maps and institutional affiliations. 